\title{
Auricular Prosthesis the Overlooked Skill in Prosthodontics: A Case Report
}

\author{
Ambuj Chandna, Abu Nedal, Tanvi Kalra, Rahul Kushwaha, Bhupinder Arora
}

\begin{abstract}
The rehabilitation of a patient with facial defects is a challenging task. An alternative to surgical reconstruction is the creation of a silicone auricular prosthesis. The replacement of anatomical parts is an art and science. These prostheses provide a cost-effective and an Acceptablemeans of camouflage for patientswho are disinclined tosurgical reconstruction. The process of creating a silicone auricular prosthesis is discussed in this article.
\end{abstract}

Keywords: Ear prosthesis,Auricular prosthesis, silicone prosthesis,maxillofacial prosthesis

\section{Introduction}

With the increased life expectancy, there are more occurrences of facial defects.

An auricular defect can be caused by any of several conditions,Postsurgical malignancies, Road side accidents (Trauma) or Congenital.

Patient presenting with anotia has an option of restoration by Surgical Reconstruction or By Prosthesis. Prosthetic options could be either Implant retained or Adhesive retained prosthesis.

These defects may be restored by reconstructive surgery but many a times due to Multiple Surgical Interventions and Compromised Esthetic Outcome Rehabilitation By Prosthetic Means Is A Chosen Option. One of the most common requisite of a facial prosthesis is that it should camouflage the defect, so that the confidence of the person is restored and that helps him to lead a normal life. Though implant retained prosthesis is a preferred option, it requires Sufficient healthy bone at the defect site for implant placement, Surgical intervention, Cost involvement and usually A time interval between implant placement and prosthetic rehabilitation.

On the contrary adhesive retained prosthesis can be placed Immediately on a healthy tissue bed, Without surgery and is Cost effective. With the advancement in technologies CAD CAM is also being used for scanning and three dimensional reconstruction of ear but it requires special armamentarium which may not freely accessible and not cost effective.

\section{Case Report}

A 28 year male patient presented with congenital anotia on the right side On examination the defect site was having healthy tissue bed with rudimentary tissue tag at the level of tragus and the left ear was normal.The patient is planned for rehabilitation with adhesive retained silicone ear prosthesis.

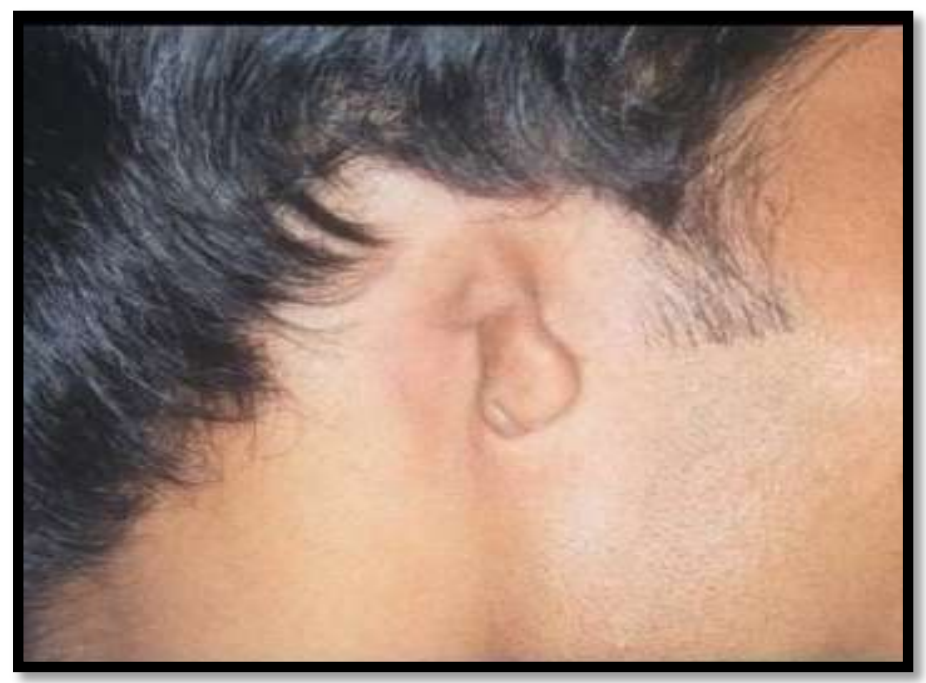

Defect site showing patient with congenitally missing ear right side. 


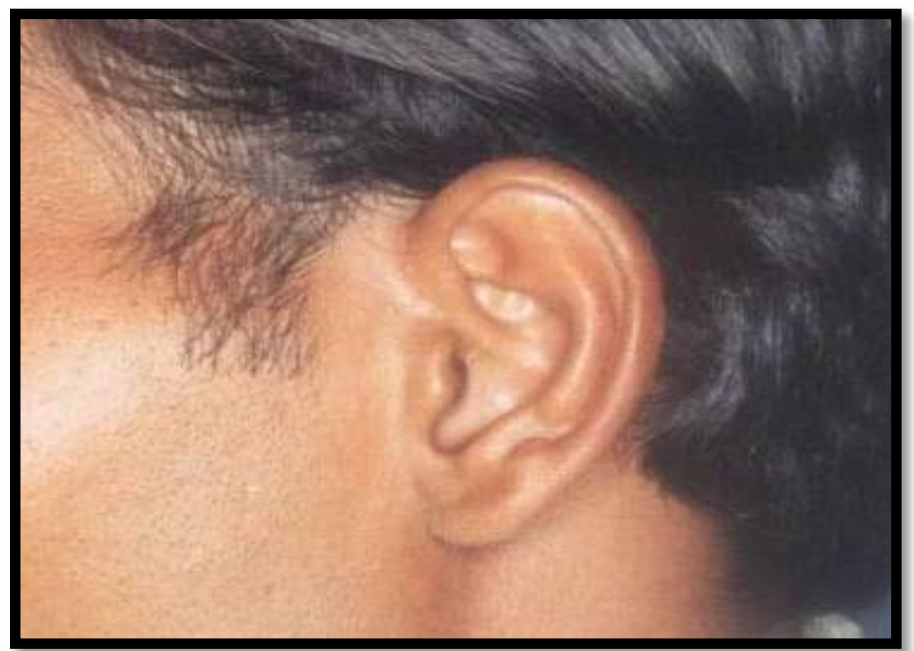

Patient with normal ear on left side.

Three horizontal markings are made on the normal ear with indelible pencil

- First at the junction of helix with the side of the head,

- Second at the mid of the tragus and

- Third at the junction of the ear lobe with the side of the head.

- These markings are copied horizontally at the same position on the defect side when the patient is looking in front.

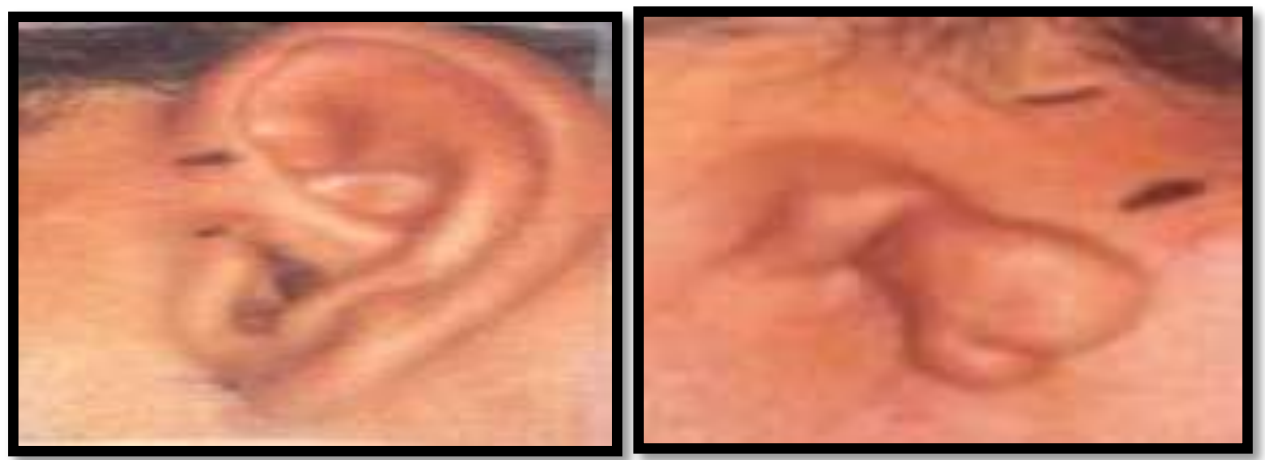

- Impression is made using alginate impression material.

Cold water is used to allow proper mix and longer working time.

Alginate is thoroughly mixed with water in 2:3 ratios and is poured without trapping of the air and the top of tray is filled with fast setting plaster to stabilize the impression.

Once the material is set the seal is break by instructing the patient to wrinkle and screw up his head and impression is carefully lifted upwards.

- The tray is place on the defect site.

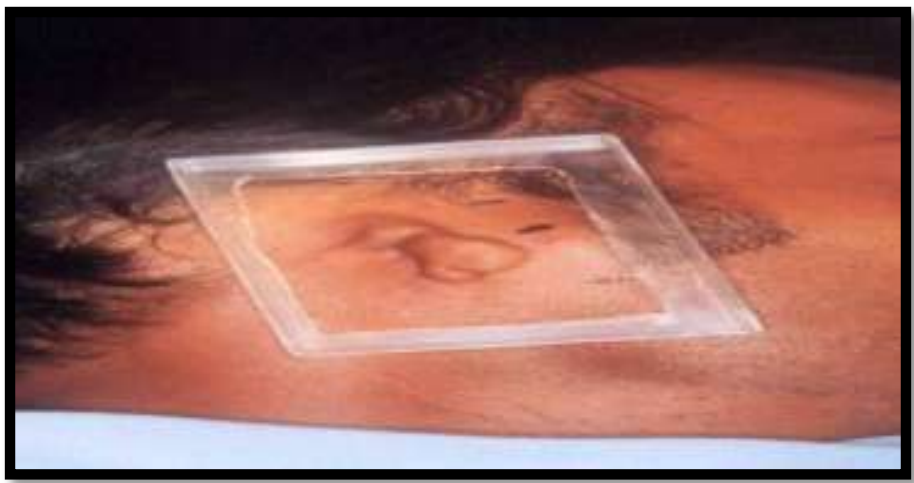

Customized tray in place defect site. 


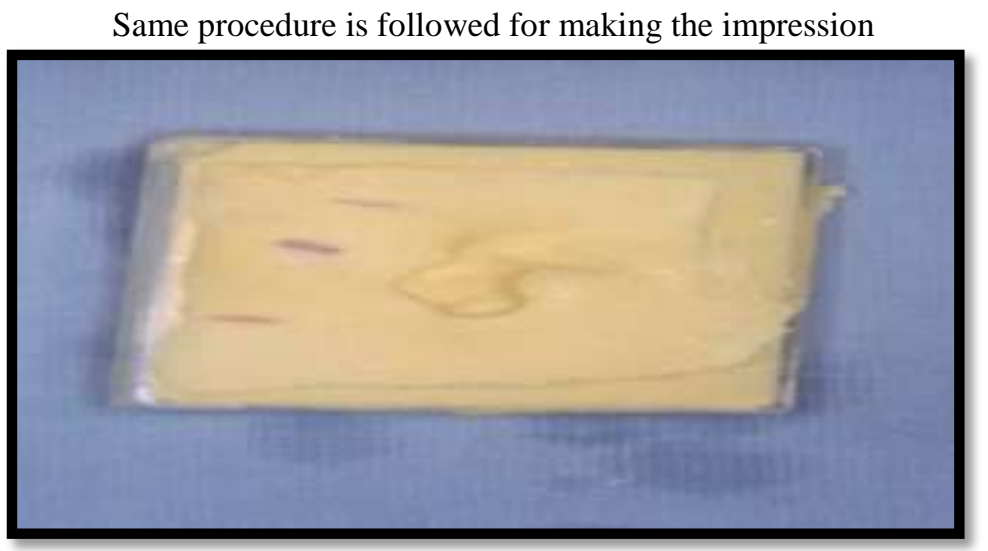

Impression of the defect site

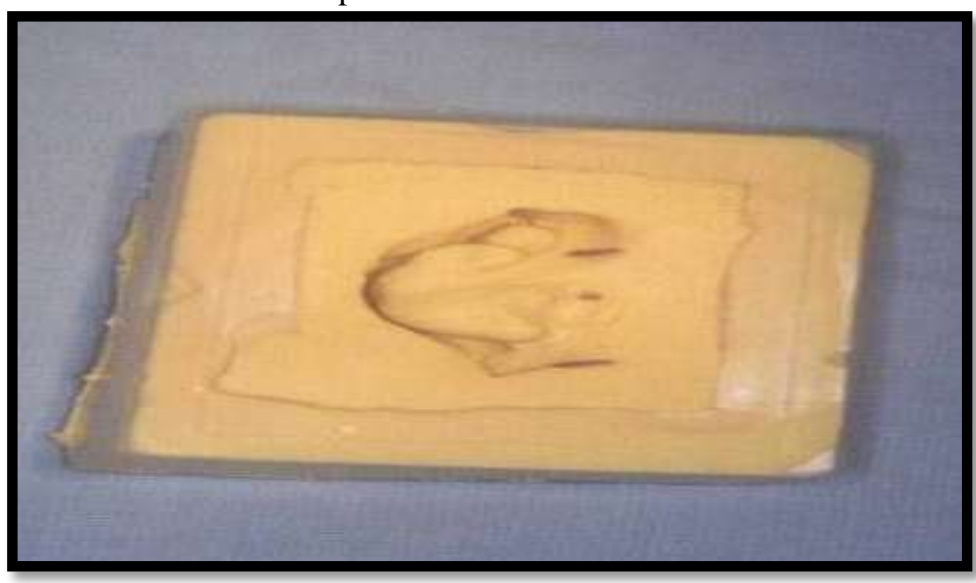

Impression of the normal ear

Now tray is boxed with modeling wax and poured with the dental stone to get the master cast of the defect site and the cast of the normal ear

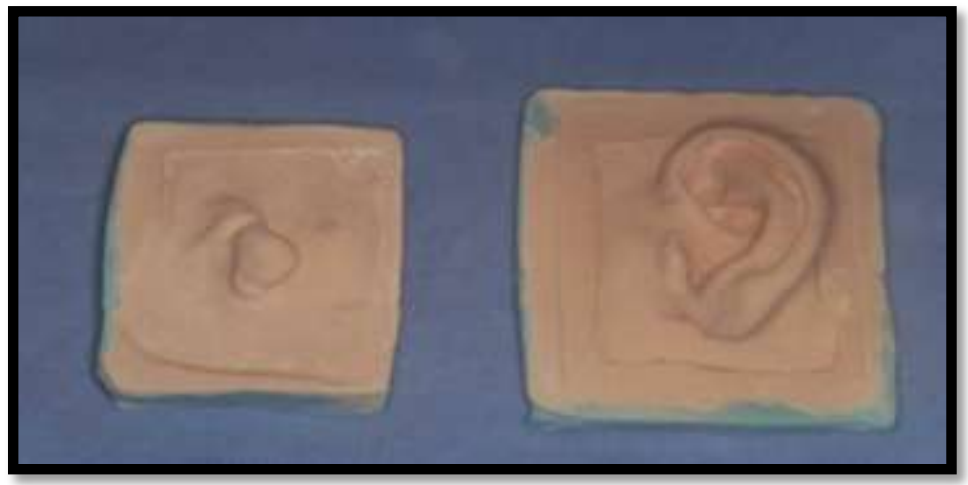

Casts of defect site and normal ear.

- Sculpting wax pattern identical to contralateral ear is a difficult task because of the complexity of the shape of an ear.

- Donor ear is a good option. It is much easier to modify the donor ear to match the patient ear.

- Selection of a donor ear can be done using ear of a sibling, brother, sister or from any person whose ear matches the normal ear of the patient.

- Once the donor ear of of a person is selected for making the pattern its impression is made and filled with modeling wax and allowed to set.

- The wax pattern is retrieved from the impression.

- The master cast of the defect site is soaked in water to prevent sticking of wax and a thin wax sheet is than adapted on the back portion of proposed ear site following the curvature of ear this will provide a base of the prosthesis and used for proper prosthesis adhesion to the tissue bed. 
- Wax ear pattern is now oriented on the master cast making use of the markings placed on the defect site and modified to match the mirror image of the contralateral ear.

- A scale is used to measure the length, width of wax pattern and a divider is used to achieve the correct projection of the wax ear pattern from the model surface

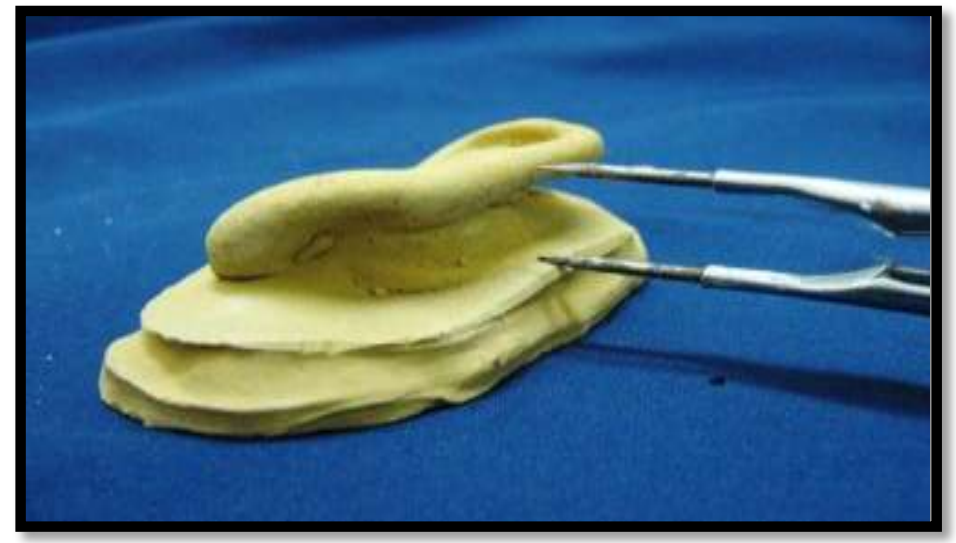

Measurement of lateral projection of normal ear.

- Necessary changes are done by observing the patient from front, side and back as the ear has a three dimensional projection and shall match with the projections of contralateral ear.

- The margins of the patterns is placed in the crevices and patient is asked to open and close the jaw and make side to side movement and confirm that the margins were not lifting on movement of the jaw if necessary required correction are made.

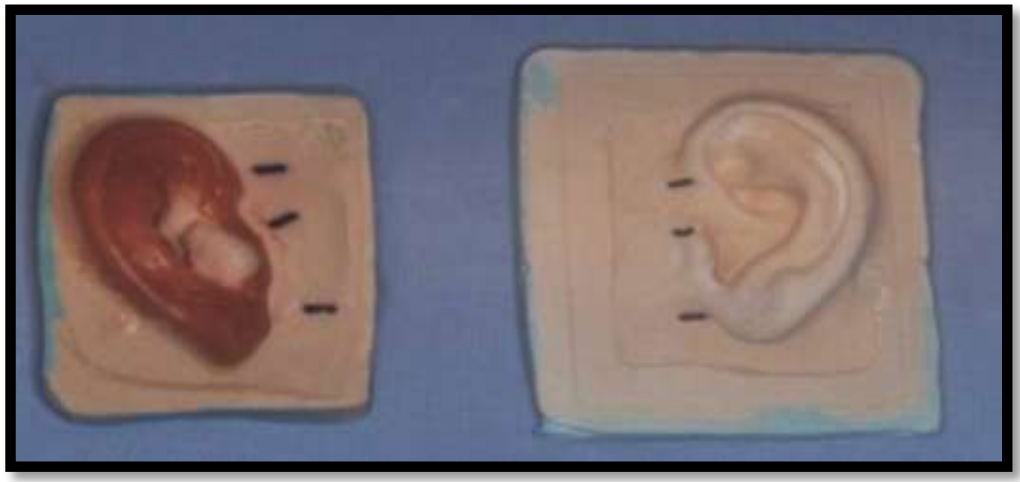

Casts of defect site with wax pattern and normal ear

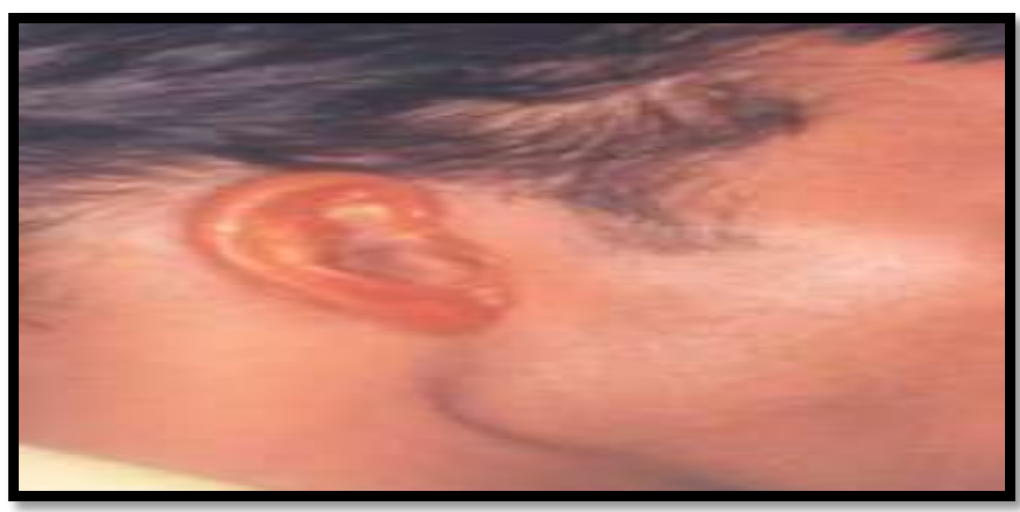

Wax pattern tried on patient in open mouth position

- The ear wax pattern is now sealed in position on the master cast and leading edges are thinned as much as possible so as to allow the silicone edges to feather in to the natural skin and when used in conjunction with adhesive they disappear.

- As the skin texture is not glossy finish, to simulate and create the tissue surface texture of ear, flaming of waxwork and damping with a wet gauge and then re-flaming is done 
- A three piece mold is fabricated for easy placement of silicone in the mold.

- To get the three piece mold, top portion of the dental flask is used as base and master cast along with the wax ear pattern is placed on this top portion of the flask and dental plaster is poured to flush with the surface of the cast leaving no undercuts.

- Once set two grooves are created on the plaster at the back portion of ear to reorient the piece of mold.

- Vaseline is applied as a separating agent and dental stone is mixed and filled on the back side of the ear wax pattern to flush just underneath the superior margin of the helix extending till base of the helix and junction of the lobe with the side of the head without leaving any undercut.

- Once this second pour is set again two grooves of different shape is created on the surface of the second part of stone mold for the reorientation of the parts and again vaseline is applied on the surface and now the body of the flask is seated on the top portion of the flask which acts as a base here and third pour is done using plaster and lid is placed and clamped and allowed to set.

- Dewaxing is done in the usual manner. After keeping the flask in hot water for fifteen minutes the flask is opened carefully and all three piece of mold are thoroughly cleaned with hot water to remove all the traces of vaseline and wax.

- Cold mold seal is diluted with water in 1:1 ratio and applied.

- The molds are allowed to dry completely as traces of vaseline, wax or water will interfere with setting of silicone and the prosthesis will have the tacky surface that will invite catching of dust at a later date.

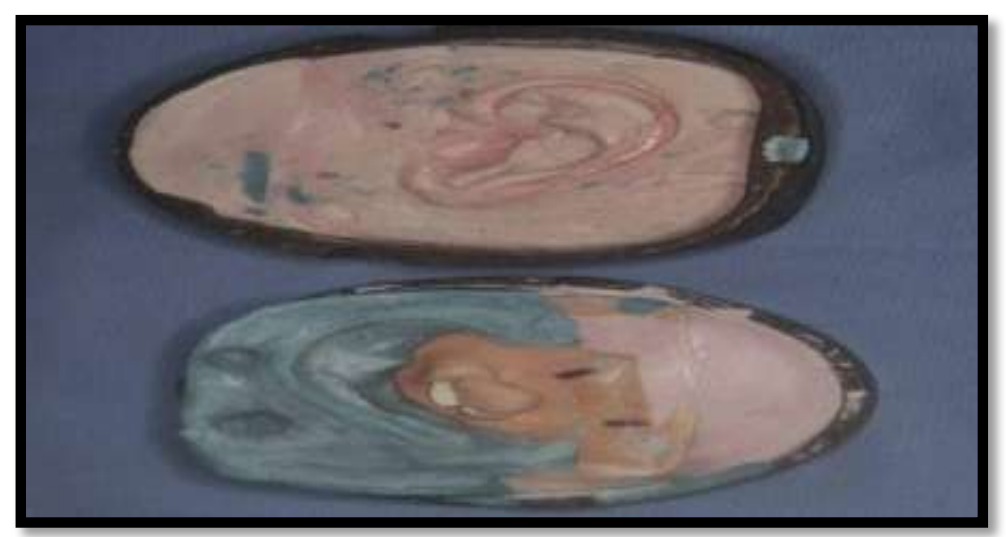

Three piece mold

- Three piece mold is now ready for silicone packing. Medical grade factor II room temperature vulcanizing (RTV) silicone is used and is mixed as per manufacturer's instruction.

- To fabricate a life like silicone prosthesis the patient must always be present for the color match

- It requires great care and patience from the doctor along with an understanding of color matching for a successful color match.

- As the silicone is translucent desired skin color can be obtained using primary colors in proper proportions.

- Using red, yellow and blue primary colors of intrinsic coloring system first base shade is prepared.

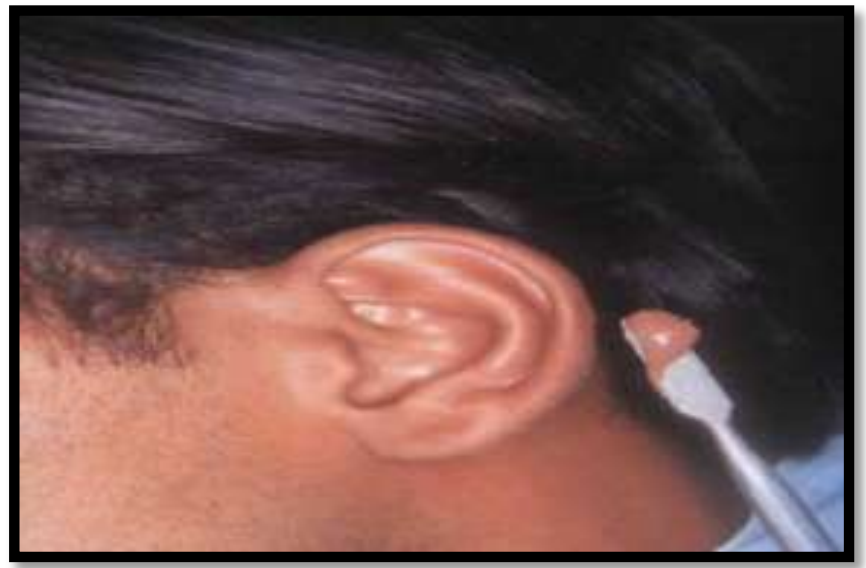

Color Matching 
- Then as per the requirement additional shades are prepared. Care is taken while adding color to get proper color, as color loading will lead to opacity and lifeless appearance to match with the patient's normal ear the dark and light shade silicone is poured in the mold.

- To create the characteristics red flocks can be place on the surface layer and mold is packed and allowed for bench curing for 24 hours.

- Cured prosthesis is retrieved from the mold, cleaned thoroughly with soap and excess silicone flesh is trimmed from the margins.

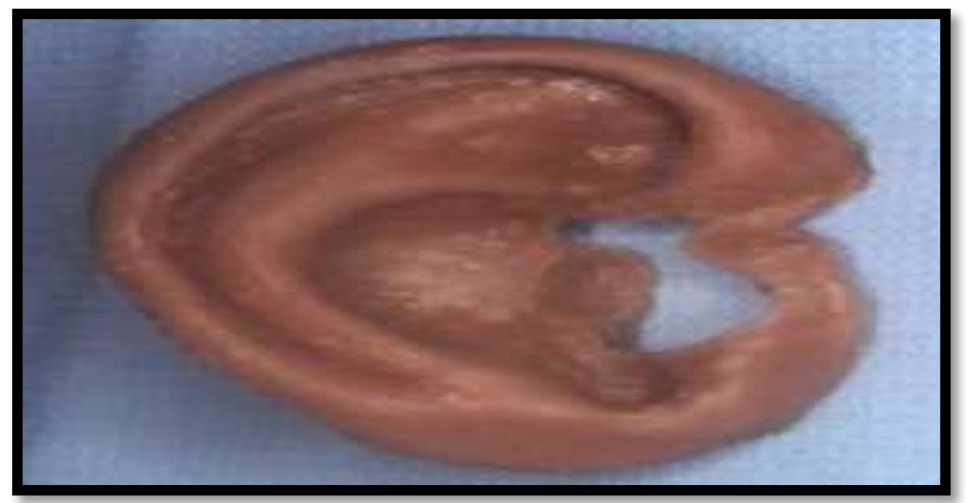

Finished silicone ear prosthesis

- Prosthesis is tried on patient and margins are trimmed as per the proposed margins.

- A thin layer of medical grade water base silicone adhesive is applied on the intaglio surface of the prosthesis and oriented on the defect and with a little pressure the prosthesis is held in place while the adhesive is cured and sets.

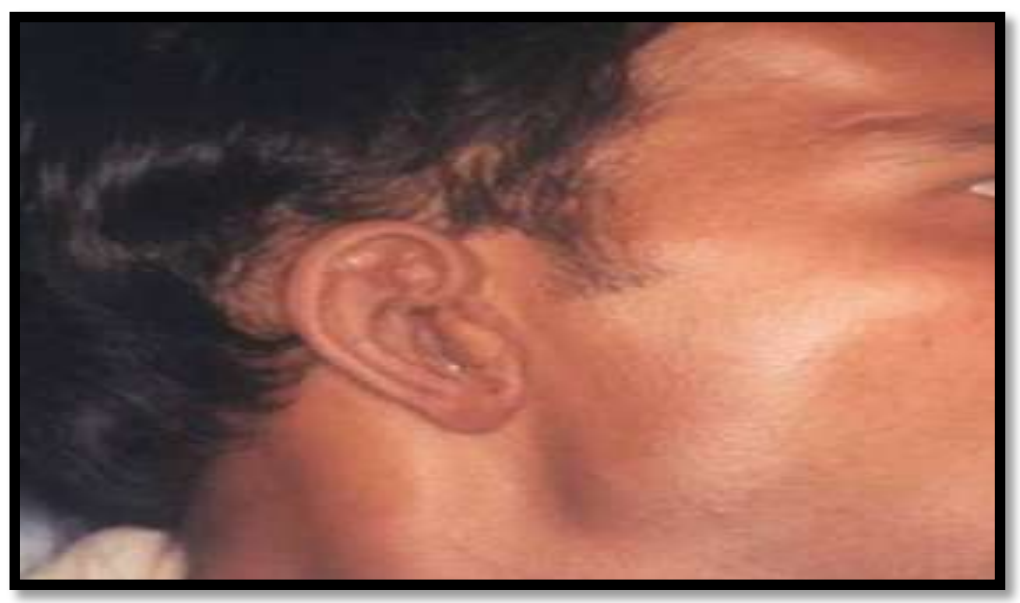

Ear prosthesis in place

- Patient is educated for placement and removal of the ear prosthesis. Home care regarding cleaning of prosthesis is also explained to the patient so as to keep tissue bed healthy. Finally the prosthesis is delivered to the patient.

\section{Discussion}

- The choice between surgical reconstruction and prosthetic restoration of facial defects is a difficult decision.

- As consistent good results have not been demonstrated in staged surgical ear reconstruction the prosthetic restoration is a preferred option.

- The application of ossiointigrated ear implants has changed patient perception about facial prosthesis because of effective retention and improved esthetics.

- Many techniques are in use for fabrication of wax pattern for adhesive retained silicone ear prosthesis. Among them

- One is SCULPTING THE PATTERN with the help of carving the wax. 
- Second is SLICING TECHNIQUE in which the wax pattern is made using slices of wax pattern of normal ear and placing them in opposite direction

- The technique used in this methodology is modification of donor ear which allows easy, quick incorporation of finer anatomical details making it more lifelike.

- With the advancement in technologies CAD CAM is also being used for scanning and three dimensional reconstruction of ear but it requires special armamentarium which may not freely accessible and not cost effective.

\section{Conclusion}

Medical grade silicone maxillofacial prosthetic material has the ability to

- Match any skin color using intrinsic and extrinsic color system and

- Are Color Stable,

- Has Rubbery Consistency to match the elasticity of skin,

- Biologically Inert thus Biocompatible.

An esthetic adhesive retained ear prosthesis is made by meticulous step by step procedure using donor ear for fabrication of wax pattern.

Rehabilitation of patient with congenital anotia is achieved by

- Restoring the defect and

- Fulfilling the objective of maintaining

1. Patient comfort,

2. Esthetics and

3. Bringing him back to the society to lead a normal life

\section{Refrences}

[1]. J. Beumer, T.A. Curtis and M.T. Marunick, Maxillofacial rehabilitation. restoration of facial defects (1 st ed. Medico Dental Media Intl, St. Louis, 1996) 385-403

[2]. K.F. Thomas, Prosthetic rehabilitation (1 st ed. Quintessence Publishing Co., Ltd., London, 1994) 55-91

[3]. T. D. Taylor, Clinical maxillofacial prosthetics (1 st ed. Quintessence Publishing Co. Inc, Illinois, 2000) 233-264

[4]. J. C. Lemon, M. S. Chambers, P. J. Wesley and J. W. Martin, Technique for fabricating a mirror-image prosthetic ear, J Prosthet Dent. 75,1996, 292-293

[5]. T. M. Kubon, Creating an adaptable anterior margin for an implant-retained auricular prosthesis, J Prosthet Dent. 86(3), 2001, 23340

[6]. P. S. Kumar, K.S. Satheesh Kumar and R.C.Savadi, Bilateral implant-retained auricular prosthesis for a patient with congenitally missing ears A clinical report, J Prosthodont, 21(2), 2012, 322-7

[7]. M.M. Hatamleh and J. Watson, Construction of an implant-retained auricular prosthesis with the aid of contemporary digital technologies clinical report, J Prosthodont, 22(2), 2013, 132-6.

[8]. E. Sivayoham and T. J. Woolford, Current opinion on auricular reconstruction, Otolaryngol Head Neck Surg, 20(4), 2012, 287-90

[9]. K. Storck , R. Staudenmaier , M. Buchberger, T. Strenger, K. Kreutzer,VonBomhard and T. Stark, Total reconstruction of the auricle: our experiences on indications and recent techniques, Biomed Res Int. 2014;373286. doi: 10.1155/2014/373286.

[10]. S. Wang, X. Leng, Y.Zheng, D. Zhang and G. Wu, Prosthesis-guided implant restoration of an auricular defect using computed tomography and 3-dimensional photographic imaging technologies: A clinical report, J Prosthet Dent. 2014; pii: S00223913(14)00425-9. doi: 10.1016/j.prosdent.2014.08.014.

[11]. M. O. Karatas, E. D. Cifter, D. O. Ozenen, A. Balik and E. B. Tuncer, Manufacturing implant supported auricular prostheses by rapid prototyping techniques, Eur J Dent. 5(4), 2011, 472-7

[12]. He Yong, Guang-huai, Xue and Fu.Jian-zhong, Fabrication of low cost soft tissue prostheses with the desktop 3D printer, 2014; doi: 10.1038/srep06973 PMCID: PMC4245596 Sci Rep. 4: 6973. 